\title{
RISCO DE CONTAMINAÇÃO DE ÁGUAS POR PESTICIDAS APLICADOS EM PLANTAÇÕES DE EUCALIPTOS E COQUEIROS: ANÁLISE PRELIMINAR
}

\author{
NATILENE MESQUITA BRITO * \\ OZELITO POSSIDÔNIO DE AMARANTE JR. ** \\ ROSÂNGELA ABAKERLI *** \\ TERESA CRISTINA RODRIGUES DOS SANTOS **** \\ MARIA LÚCIA RIBEIRO *****
}

\begin{abstract}
Efetuou-se avaliação preliminar de pesticidas aplicados em plantações de eucaliptos e coqueiros no Nordeste brasileiro quanto ao risco de contaminação de águas superficiais e subterrâneas. Para tanto, foram utilizados os critérios sugeridos pela Agência de Proteção Ambiental dos Estados Unidos (USA), o índice "Growndwater Ubiquity Score" (GUS) e o método de Goss. Os pesticidas Tetradifon, Triclorfon, $\alpha$-Endosulfan, $\beta$-Endosulfan, Sulfato de Endosulfan e Glifosato apresentaram potencial de contaminação das águas que exige monitoramento. Segundo os critérios avaliados neste estudo, os demais pesticidas investigados não apresentaram risco de contaminação.
\end{abstract}

PALAVRAS-CHAVE: ÁGUA-CONTAMINAÇÃO; CONTAMINAÇÃO-RISCOS; ÁGUAS SUBTERRÂNEAS; ÁGUAS SUPERFICIAIS.

* Pós-Graduanda em Química, Instituto de Química, Universidade Estadual de São Paulo (UNESP), Araraquara, SP. (e-mail: natibrit@posgrad.iq.unesp.br).

** Pós-Graduando em Química, Departamento de Tecnologia Química (DTQ), Centro de Ciências Exatas e Tecnologia, Universidade Federal do Maranhão (UFMA), Campus do Bacanga, São Luís, MA. (e-mail: ozelito@terra.com.br).

*** Técnica da EMBRAPA Meio Ambiente, Jaguariúna, SP.

**** Professora, Doutora em Química, DTQ, Centro de Ciências Exatas e Tecnologia, UFMA, Campus do Bacanga, São Luís, MA.

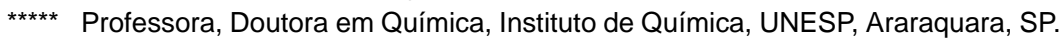




\section{INTRODUÇÃO}

A principal estratégia no combate e prevenção de pragas agrícolas tem sido o intenso uso de pesticidas (1). Tal necessidade é atribuída à homogeneização do ambiente, o que transforma muitos predadores naturais em pragas (2). Durante a última década, a quantidade e variedade de pesticidas aplicados em frutas e vegetais aumentou verticalmente (US\$31,2 bilhões em 1996). Com o intuito de monitorar eventuais resíduos de pesticidas no ambiente e em alimentos, métodos analíticos foram desenvolvidos para avaliar o risco de contaminação do solo e das águas subterrâneas e superficiais (3-6). O monitoramento das águas subterrâneas tem assumido grande importância nos últimos anos, sendo realizado, principalmente, em locais cujas águas subterrâneas constituem fontes primárias de água potável e em áreas próximas a regiões agrícolas, nas quais os pesticidas causam contaminação nos sistemas hidrológicos. Estes programas de monitoramento enfocam as classes de pesticidas mais utilizadas considerando as quantidades de aplicação e a produção agrícola (7).

Efetuou-se, neste trabalho, análise preliminar dos riscos de contaminação das águas subterrâneas e superficiais por alguns pesticidas das classes dos organoclorados (OC), organofosforados (OF), clorofenoxiácidos e glicinas, comumente utilizados nas culturas de coqueiros e eucaliptos.

Os organoclorados apresentam a característica de serem lipossolúveis e, conseqüentemente, podem se acumular em organismos vivos, além de persistirem por muito tempo no ambiente. Muitos compostos organoclorados são proibidos e os demais pesticidas desta classe têm sido aplicados como inseticidas em diversas culturas (8).

Os pesticidas organofosforados representam classe importante de inseticidas, geralmente, derivados dos ácidos fosfórico e tiofosfórico (9). Os organofosforados apresentam alta toxicidade e sua ação no organismo se processa pela inibição da enzima colinesterase com conseqüente acúmulo da acetilcolina nas fibras nervosas. Assim, impede a transmissão de novos impulsos e gera uma série de efeitos no organismo tais como convulsões, paradas respiratórias e coma $(10,11)$.

Os pesticidas clorofenoxiácidos constituem importante classe de herbicidas (12). Estes herbicidas normalmente apresentam longo tempo de atividade residual em solos e águas persistindo por vários meses devido à sua baixa atividade microbiológica (13). Entretanto, alguns herbicidas da classe dos ácidos fenóxidos com baixa persistência permanecem no solo por, aproximadamente, 2 semanas após a aplicação. Os herbicidas desta classe degradam-se facilmente em água, por ação da luz solar e 
de microorganismos (14). O ácido 2,4-diclorofenoxiacético (2,4-D), membro desta classe, é altamente seletivo, sistêmico e pós-emergente (15).

O Glifosato pertence à classe das glicinas, sendo considerado de baixa toxicidade aguda (15) devido à modalidade bioquímica de ação do herbicida. Este composto age através de um caminho metabólico nas plantas, similar ao existente em alguns microorganismos mais complexos, mas inexistente em animais. O Glifosato pode, no entanto, impedir a ação de funções enzimáticas nos animais. Quando injetado no abdômen de ratos causou diminuição da atividade de algumas enzimas (16).

A intensidade do uso de pesticidas e os efeitos que causam ao ambiente e à saúde humana exigem o estudo de suas principais propriedades físico-químicas. Além disso, deve-se prever suas interações com o solo e a possibilidade de contaminação e transporte, quando dissolvidos em água ou associados aos sedimentos. A necessidade da avaliação do risco de contaminação se deve ao fato de que a agricultura exige considerável suprimento de água, o que conduz ao desenvolvimento desta atividade próximo a rios e lagos (2).

O potencial de contaminação de águas superficiais e subterrâneas tem sido determinado, preliminarmente, mediante avaliação teórica das características físico-químicas dos compostos aplicados, bem como das propriedades do solo (17-19). Três procedimentos têm sido amplamente utilizados para estas avaliações, ou seja, critérios de "Screening" da Agência de Proteção Ambiental dos Estados Unidos (US-EPA); índice de vulnerabilidade de águas subterrâneas (Groundwater Ubiquity ScoreGUS); e o método de Goss. Neste trabalho foram empregados estes índices para avaliar quais dos pesticidas estudados podem exigir investigações quanto à presença de resíduos no ambiente.

\section{MATERIAL E MÉTODOS}

Efetuou-se levantamento bibliográfico sobre os pesticidas aplicados nas culturas de eucaliptos e coqueiros e das propriedades físico-químicas dos princípios ativos. Em seguida, foram aplicados modelos que possibilitam a avaliação preliminar do risco potencial em função das características das substâncias usadas. Os critérios da EPA envolvem os valores de solubilidade em água (a $\left.25^{\circ} \mathrm{C}\right)$, o coeficiente de adsorsão à matéria orgânica do solo (Koc), a constante da Lei de Henry $(\mathrm{KH})$, a especiação (presença de forma aniônica em pH normal, entre 5 e 8) e a meia-vida no solo e na água (DT50) (Quadro 1). O índice GUS inclui o valor de meia-vida do composto no solo (DT50) e o coeficiente de adsorsão deste composto à matéria orgânica do solo (Koc). Já o método de Goss 
considera a meia-vida do composto no solo (DT50), sua solubilidade em água (a $25^{\circ} \mathrm{C}$ ) e a constante de adsorsão à matéria orgânica do solo (Koc).

\section{QUADRO 1 - CRITÉRIOS DE AVALIAÇÃO EPA E GUS}

\begin{tabular}{|c|c|}
\hline \multicolumn{2}{|c|}{ CritØrio EPA } \\
\hline Solubilidade em Agua & $>30 \mathrm{mg} / \mathrm{L}$ \\
\hline Koc & $<300-500$ \\
\hline $\mathrm{K}_{\mathrm{H}}$ & $<10^{-2} \mathrm{~Pa} \cdot \mathrm{m}^{3} / \mathrm{mol}$ \\
\hline Especia $\stackrel{\text { a }}{0}$ & Negativamente carregado \\
\hline $\mathrm{DT}_{50}$ no solo & $>2-3$ semanas \\
\hline $\mathrm{DT}_{50}$ na Ftgua & $>25$ semanas \\
\hline \multicolumn{2}{|c|}{ CritØrio GUS } \\
\hline $\mathrm{N}^{\mathrm{a}}$ o sofre lixivia $\mathrm{a}$ & $<1,8$ \\
\hline Faixa de transi $\stackrel{a}{0}$ & $1,8<\mathrm{GUS}<2,8$ \\
\hline ProvÆ el lixivia & $>2,8$ \\
\hline
\end{tabular}

Os critérios propostos para a avaliação do potencial de contaminação pelo método de Goss são:

- Alto potencial de transporte associado ao sedimento (APTAS) - DT50 no solo $\geq 40$ dias e $\mathrm{Koc}=1000$, ou DT50 no solo $\geq 40$ dias, $\mathrm{Koc} \geq 500$ e solubilidade em água $(\mathrm{S})=0,5 \mathrm{mg} / \mathrm{L}$.

- Baixo potencial de transporte associado ao sedimento (BPTAS) - DT50 no solo $<1$ dia, ou DT50 $\leq 40$ dias, Koc $\leq 500$ e S $\geq 0,5 \mathrm{mg} / \mathrm{L}$, ou DT50 no solo $\leq 40$ dias, $\mathrm{Koc} \leq 900$ e $S \geq 2 \mathrm{mg} / \mathrm{L}$, DT50 no solo $\leq 2$ dias e $\mathrm{Koc} \leq 500$, ou DT50 no solo $\leq 4$ dias, $\mathrm{Koc} \leq 900$ e $S \geq 0,5 \mathrm{mg} / \mathrm{L}$.

- Alto potencial de transporte dissolvido em água (APTDA) - DT50 no solo $>35$ dias, $\mathrm{Koc}<1.000 .000$ e $\mathrm{S} \geq 1 \mathrm{mg} / \mathrm{L}$, ou DT50 no solo $>35$ dias, $\mathrm{Koc} \leq 700$ e $10 \leq \mathrm{S} \leq 100 \mathrm{mg} / \mathrm{L}$.

- Baixo potencial de transporte dissolvido em água (BPTDA) $K_{o c} \geq 1.000 .000$, ou DT50 no solo $\leq 1$ dia e Koc $\leq 100$, ou DT50 no solo $<35$ dias e $\mathrm{S}<0,5 \mathrm{mg} / \mathrm{L}$. 
A Figura 1 evidencia como os critérios de GOSS se relacionam devendo-se avaliar as características da substância e suas combinações. As substâncias com potencial médio para contaminarem águas superficiais não se enquadram nos critérios anteriormente citados.

\section{FIGURA 1 - CRITÉRIOS DE AVALIAÇÃO DO MÉTODO DE GOSS}

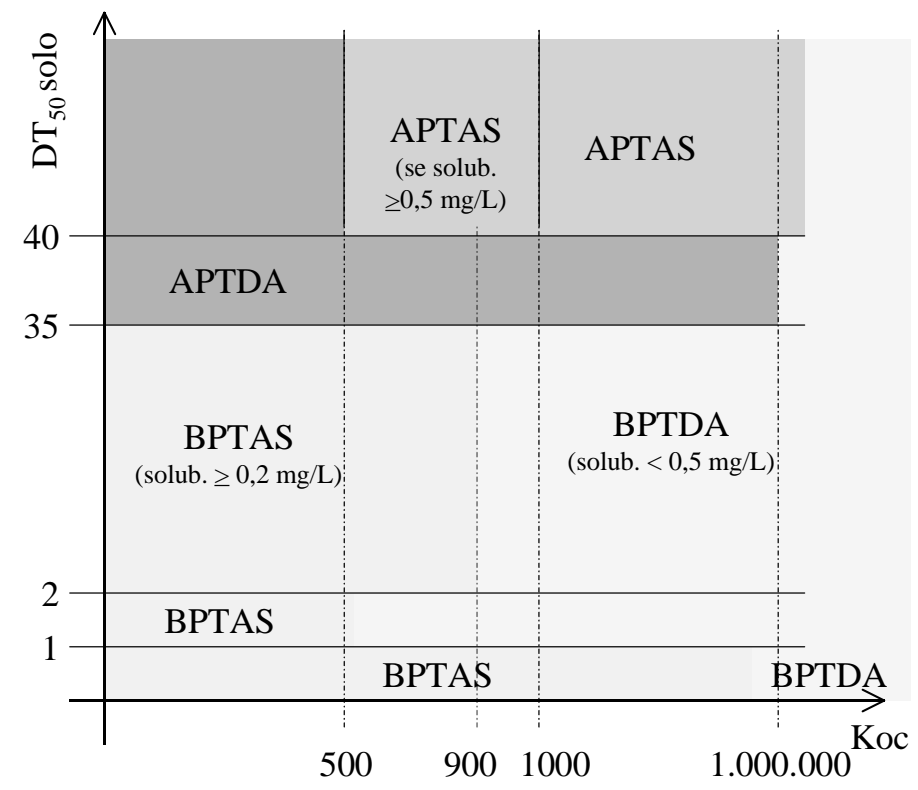

\section{RESULTADOS E DISCUSSÃO}

\subsection{PROPRIEDADES DOS COMPOSTOS ESTUDADOS}

Foram selecionados os seguintes pesticidas: Ácido 2,4Diclorofenoxiacético (2,4-D) e Glifosato (eucaliptocultura), Malation, Captan, Paration-metil, $\alpha$-Endosulfan, $\beta$-Endosulfan, Sulfato de Endosulfan, Monocrotofós, Tetradifon e Triclorfon (cocoicultura). A Figura 2 apresenta as fórmulas moleculares dos compostos selecionados e suas propriedades físico-químicas constam da Tabela 1.

Os resultados referentes aos compostos individuais são apresentados na Tabela 2. 
FIGURA 2 - FÓRMULA ESTRUTURAL DOS COMPOSTOS ESTUDADOS

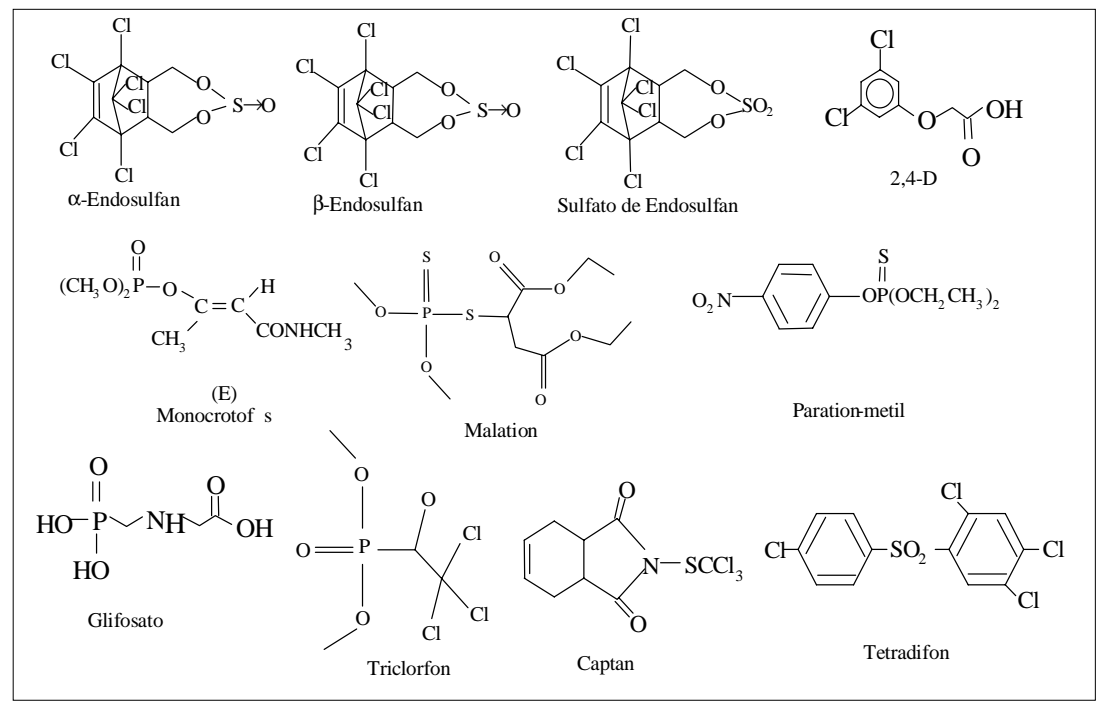

\section{TABELA 1 - PROPRIEDADES FÍSICO-QUÍMICAS DOS PRINCÍPIOS ATIVOS}

\begin{tabular}{l|c|c|c|c|c|c|c|c}
\hline Composto & DT $_{50 \text { solo }}$ & DT50fgua & $\begin{array}{c}\text { Solub. } \\
\text { Agua } \\
\text { (mg/L) }\end{array}$ & Especia a o & Koc & LogKow & $\mathrm{K}_{\mathrm{H}}$ & Volatilidade \\
\hline Captan & 2 & 2 & 3,3 & - & 200 & 2,78 & - & \\
$2,4-\mathrm{D}$ & 7 & 1 & 311 & Negativo & 60 & 2,723 & $1,4.10^{-9}$ & $3,4.10^{-1}$ \\
$\alpha$-endosulfan & 35 & 28 & 0,32 & - & 3,89 & 4,74 & - & $9.10^{-3}$ \\
$\beta$-endosulfan & 150 & 28 & 0,33 & - & 3,89 & 4,79 & - & $9.10^{-6}$ \\
Sulfato de & 77 & - & 0,22 & - & 3,89 & - & - & - \\
endosulfan & & & & & & & & \\
Glifosato & 60 & 30 & 12000 & Ambos & 11275 & $-3,58$ & $2,5.10^{-6}$ & $3.10^{-7}$ \\
Monocrotof s & 3 & 131 & 1000 & - & - & $-0,22$ & - & $7.10^{-6}$ \\
Malation & 13 & 21 & 145 & - & 1800 & 2,75 & - & $4.10^{-5}$ \\
Paration metil & 33 & 9 & 55 & - & 5100 & 3,709 & - & $9,7.10^{-6}$ \\
Tetradifon & 252 & - & 80 & - & - & 4,61 & - & $7,5.10^{-3}$ \\
Triclorfon & 15 & 1 & 154 & - & 10 & 5,75 & - & $7,8.10^{-6}$ \\
& & & & & & & & \\
\hline
\end{tabular}


O Índice GUS avalia o potencial de determinado composto ser lixiviado, atingindo águas subterrâneas, por meio das propriedades do próprio princípio ativo, desconsiderando as propriedades do solo. O método de Goss foi utilizado para discutir as possibilidades dos pesticidas atingirem as águas superficiais e o seu potencial de transporte, quer dissolvido, quer associado ao sedimento.

\section{TABELA 2 - RESULTADO INDIVIDUAL DA AVALIAÇÃO DE RISCO DOS PESTICIDAS ESTUDADOS}

\begin{tabular}{l|c|c|c}
\hline Composto & GUS & GOSS & EPA \\
\hline CAPTAN & BL & BPTAS & $\mathrm{N}$ \\
$2,4-D$ & $\mathrm{ML}$ & BPTAS & $\mathrm{P}$ \\
ENDOSULRAN $\alpha$ & $\mathrm{PL}$ & BPTDA & $\mathrm{PP}$ \\
ENDOZULFAN $\beta$ & $\mathrm{PL}$ & BPTDA & $\mathrm{PP}$ \\
SULFATO DE ENDOSULFAN & $\mathrm{PL}$ & BPTDA & $\mathrm{P}$ \\
GLIFOSATO & $\mathrm{BL}$ & APTAS & $\mathrm{PP}$ \\
MONOCROTOF S & $\mathrm{DI}$ & MPTA & $\mathrm{PP}$ \\
MALATION & $\mathrm{BL}$ & MPTA & $\mathrm{N}$ \\
PARATION METIL & $\mathrm{BL}$ & MPTA & $\mathrm{PP}$ \\
TETRADIFON & $\mathrm{DI}$ & APTDA & $\mathrm{P}$ \\
TRICLORFON & $\mathrm{PL}$ & BPTAS & $\mathrm{P}$ \\
\hline
\end{tabular}

BPTAS = Baixo potencial de transporte em águas no sedimento.

APTAS = Alto potencial de transporte associado ao sedimento.

APTDA = Alto potencial de transporte dissolvido na água.

MPTA = Médio potencial de transporte em águas.

$\mathrm{PL}=$ Provável Lixiviação; $\mathrm{BL}=$ Baixa Lixiviação.

$\mathrm{ML}=$ Potencial mediano para lixiviação.

$\mathrm{N}=$ Negativo.

$\mathrm{P}=$ Positivo.

$\mathrm{PP}=$ Parcialmente positivo.

$\mathrm{DI}=$ Dados Insuficientes.

Para os compostos Tetradifon e Monocrotofós, a ausência de dados de Koc na literatura consultada impossibilitou a avaliação pelo índice GUS. Para os demais compostos, os resultados sugerem que $\alpha$-Endosulfan, $\beta$ Endosulfan, Sulfato de Endosulfan e Triclorfon apresentam possibilidade de lixiviação, podendo contaminar águas subterrâneas. Os pesticidas Captan, Glifosato, Malation e Paration metil não sofrem lixiviação devendopermanecer imobilizados no solo. O pesticida 2,4-D enquadrouse na faixa intermediária para o índice de GUS (Figura 3). 
Quanto ao método de Goss, o herbicida Glifosato apresentou alto potencial de transporte associado ao sedimento, enquanto que o inseticida Tetradifon evidenciou alto potencial de transporte dissolvido na água. Os demais compostos não apresentaram riscos de contaminação das águas superficiais, segundo este método (Figura 4).

\section{FIGURA 3 - RESULTADO INDIVIDUAL DO ÍNDICE GUS PARA OS PESTICIDAS ESTUDADOS}

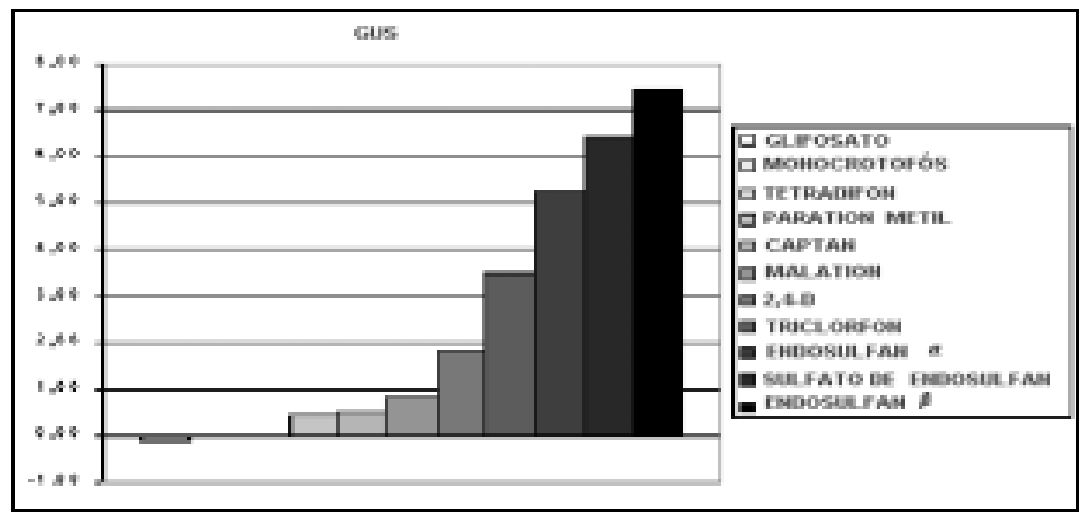

\section{FIGURA 4 - RESULTADOS INDIVIDUAIS PARA OS PESTICIDAS ESTUDADOS SEGUNDO O MÉTODO DE GOSS}

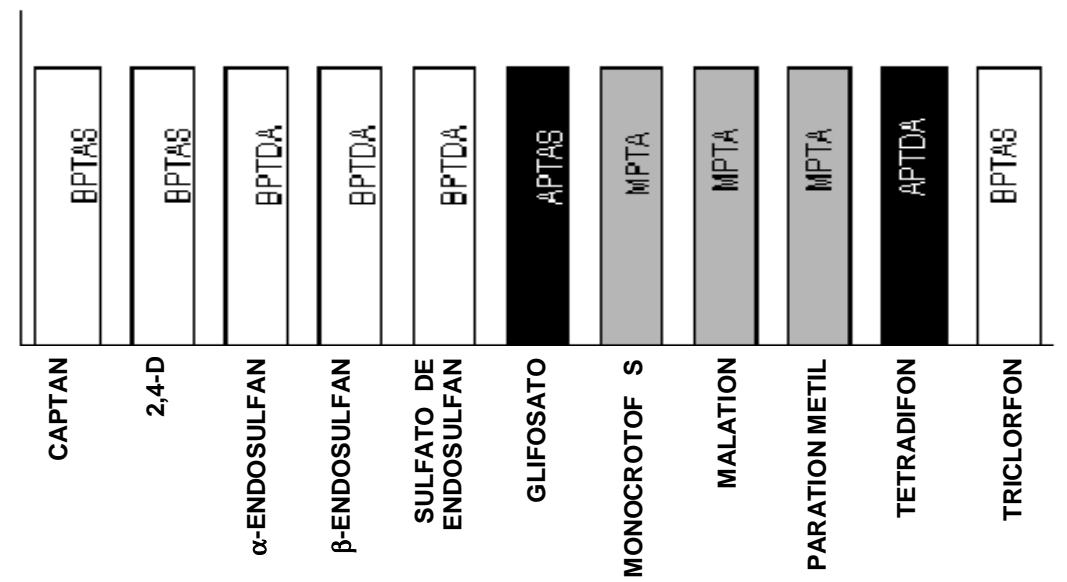


De acordo com os critérios sugeridos pela EPA, os pesticidas 2,4$D$, Sulfato de Endosulfan, Tetradifon e Triclorfon revelaram considerável risco de contaminação de águas subterrâneas. Os compostos $\alpha$-Endosulfan, $\beta$-Endosulfan, Glifosato, Monocrotofós e Paration metil apresentam resultados positivos para alguns desses critérios. Por outro lado, os pesticidas Captan e Malation não mostraram tendência para contaminação destas águas.

Deve-se ressaltar, entretanto, que os critérios aqui avaliados dizem respeito unicamente ao comportamento do pesticida no ambiente. Outros parâmetros, no entanto, precisam ser considerados, entre os quais se destacam: condições de aplicação, índice pluviométrico, características do solo e temperatura. Estes parâmetros, obviamente, podem concorrer com as propriedades físico-químicas para aumentar o potencial inerente ao pesticida, acarretando contaminação das águas subterrâneas ou superficiais.

\section{CONCLUSÃO}

Considerando as propriedades físico-químicas dos princípios ativos estudados, o risco de contaminação de águas superficiais e subterrâneas, nas áreas em que são aplicados não pode ser desprezado para os pesticidas Tetradifon, Triclorfon, $\alpha$-Endosulfan, $\beta$-Endosulfan, Sulfato de Endosulfan e Glifosato que exigem programas de monitoramento. Destes, o Glifosato apresenta maior risco de contaminação das águas superficiais quando associado ao sedimento. É possível que este pesticida seja encontrado ligado ao material em suspensão nas amostras de água provenientes de locais em que tenha sido aplicado diretamente para controle de ervas aquáticas. Entretanto, a contaminação de lençóis subterrâneos por Glifosato é pouco provável, pois não sofre lixiviação, devido à forte interação deste composto com o solo. Segundo os critérios avaliados neste estudo, os demais pesticidas investigados não apresentaram risco de contaminação.

\section{Abstract}

PESTICIDES APPLIED IN EUCALYPTUS AND COCONUT PALM PLANTATIONS: PRELIMINARY ANALYSIS

A preliminary evaluation of pesticides applied in eucalyptus and coconut crops in Northeast Brazil was carried out on the contamination risk of ground and superficial waters. For such, it was used the criteria suggested by the United States Environmental Protect 
Agency (EPA) and the "Groundwater Ubiquity Score" (GUS), and the Goss method. The pesticides Tetradiphon, Triclorphon, $\alpha$-Endosulfan, $\beta$-Endosulfan, Endosulfan Sulfate and Glyphosate showed considerable potential contamination of waters, which need monitoring. The other pesticides researched didn't show risk of contamination by following the criteria evaluated in this study.

KEY-WORDS: WATER CONTAMINATION; CONTAMINATION RISKS; GROUNDWATER; SUPERFICIAL WATER.

\section{REFERÊNCIAS}

1 CALDAS, E.D.; SOUZA, L. C. de. Avaliação de risco crônico da ingestão de resíduos de pesticidas na dieta brasileira. R. Saúde Pública, São Paulo, v. 34, n. 5, p. 529-537, 2000.

2 CARVALHO, A.R.; SCHLITTLER, F.H.M.; TORNISIELO, V.L. Relações da atividade agropecuária com parâmetros físico-químicos da água. Química Nova, São Paulo, v. 23, n. 5, p. 618-622, 2000.

3 AGUERA, A.; CONTRERAS, M.; FERNANDEZ-ALBA, A. R. Gas chromatographic analysis of organophosphorus pesticides of horticultural concern. J. Chromatogr. A., v. 655, n. 1-2, p. 293-300, 1993.

4 GOODRICH, J.A.; LYKINS Jr., B.W.; CLARCK, R.M. Drinking water from agriculturally contaminated ground water. J. Environ. Qual., v. 20, n.4, p. 707-717, 1991.

5 TESADA, A.W. Pesticide residues in foods and the environment as a consequence of crop protection. Philippin. Agricult., v. 78, n. 1, p. 63-79, 1995.

6 PEREIRA, W. E.; HOSTETTLER, F. D. Nonpoint source contamination of true Mississipi River and its tributaries by herbicides. Environ. Sci. Technol., v. 27, n. 8, p. 1542-1552, 1993.

7 FERNANDEZ-ALBA, A. R.; AGUERA, A.; CONTRERAS, M.; PEÑUELA, G.; FERRER, I.; BARCELÓ, D. Comparison of various sample handling and analytical procedures for the monitoring of pesticides and metabolites in ground waters. J. Chromatogr. A., v. 823, n. 1-2, p. 35-47, 1998. 
8 MARONI, M.; COLOSIO, C.; FERIOLI, A.; FAIT, A. Organochlorine pesticides. Toxicology, Amsterdã, v. 143, n. 1, p. 61-75, 2000.

9 MARONI, M.; COLOSIO, C.; FERIOLI, A.; FAIT, A. Organophosphorus pesticides. Toxicology, Amsterdã, v. 143, n. 1, p. 9-37, 2000.

10 SULTATOS, L. G. Mammalian toxicology of organophosphorus pesticides. J. Toxicol. Environ. Health, v. 43, n. 3, p. 271-289, 1994.

11 ENVIRONMENTAL Health Criteria: glyphosate. Genebra: World Health Organization, 1986. v. 63.

12 SANCHEZ-BRUNETE, C.; GARCIA-VALCARCEL, A. I.; TADEO, J. $L$. Determination of residues of phenoxi acid herbicides in soil and cereals by gas chromatography-ion trap detection. J. Chromatogr. A., v. 675 , n. 1-2, p. 213-218, 1994.

13 SANTOS, T.C.R.; ROCHA, J. C. Determination of rice herbicides, their transformation products and clofibric acid using on-line solidphase extraction followed by liquid chromatography with diode array and atmospheric pressure chemical ionization mass spectrometric detection. J. Chromatogr. A., Amsterdã, v. 879, n. 1-2, p. 3-12, 2000.

14 KAMRIN, M. A. Pesticides profile: toxicity, environmental impact, and fate. Nova York: Lewis, [1990?]. p. 299-309.

15 TOMLIN, C. (Ed.). The pesticide manual: incorporating the agrochemicals handbook. $10^{\text {th }}$ ed. Cambridge: British Crop Protection Council, the Royal Society of Chemistry, 1994. 1341 p.

16 GLYPHOSATE: fact sheet. Disponível em: <http://www.gn.apc.org/ pesticidestrust/aifacts/glyphosa.htm>. Acesso em 19 fev. 2000.

17 VAN DER WERF, H.M.G.; ZIMMER, C. An indicator of pesticide environmental impact based on a Fuzzy expert system. Chemosphere, v. 36, n. 10, p. 2225-2249, 1998. 
18 ROUSSEL, O.; CAVELIER, A.; VAN DER WERF, H.M.G. Adaptation and use of a Fuzzy expert system to assess the environmental effect of pesticides applied to field crops. Agriculture, Ecosystems and Environment, v. 80, n. 1-2, p. 143-158, 2000.

19 KREUGER, J.; TORNQVIST, L. Multiple regression analysis of pesticide occurrence in stream-flow related to pesticide properties and quantities applied. Chemosphere, Amsterdã, v. 37, n. 2, p. 189-207, 1998. 